\title{
The Italian National Seismic Network and the earthquake and tsunami monitoring and surveillance systems
}

\author{
Alberto Michelini, Lucia Margheriti, Marco Cattaneo, Gianpaolo Cecere, Giuseppe D'Anna, Alberto Delladio, \\ Milena Moretti, Stefano Pintore, Alessandro Amato, Alberto Basili, Andrea Bono, Paolo Casale, Peter Danecek, \\ Martina Demartin, Licia Faenza, Valentino Lauciani, Alfonso Giovanni Mandiello, Alessandro Marchetti, \\ Carlo Marcocci, Salvatore Mazza, Francesco Mariano Mele, Anna Nardi, Concetta Nostro, Maurizio Pignone, \\ Matteo Quintiliani, Sandro Rao, Laura Scognamiglio, and Giulio Selvaggi
}

Istituto Nazionale di Geofisica e Vulcanologia, Centro Nazionale Terremoti, Rome, 00143, Italy

Correspondence to: Lucia Margheriti (lucia.margheriti@ingv.it)

Received: 15 July 2016 - Revised: 11 October 2016 - Accepted: 14 October 2016 - Published: 9 November 2016

\begin{abstract}
The Istituto Nazionale di Geofisica e Vulcanologia (INGV) is an Italian research institution, with focus on Earth Sciences. INGV runs the Italian National Seismic Network (Rete Sismica Nazionale, RSN) and other networks at national scale for monitoring earthquakes and tsunami as a part of the National Civil Protection System coordinated by the Italian Department of Civil Protection (Dipartimento di Protezione Civile, DPC).
\end{abstract}

RSN is composed of about 400 stations, mainly broadband, installed in the Country and in the surrounding regions; about 110 stations feature also co-located strong motion instruments, and about 180 have GPS receivers and belong to the National GPS network (Rete Integrata Nazionale GPS, RING).

The data acquisition system was designed to accomplish, in near-real-time, automatic earthquake detection, hypocenter and magnitude determination, moment tensors, shake maps and other products of interest for DPC. Database archiving of all parametric results are closely linked to the existing procedures of the INGV seismic monitoring environment and surveillance procedures. INGV is one of the primary nodes of ORFEUS (Observatories \& Research Facilities for European Seismology) EIDA (European Integrated Data Archive) for the archiving and distribution of continuous, quality checked seismic data. The strong motion network data are archived and distributed both in EIDA and in event based archives; GPS data, from the RING network are also archived, analyzed and distributed at INGV.

Overall, the Italian earthquake surveillance service provides, in quasi real-time, hypocenter parameters to the DPC.
These are then revised routinely by the analysts of the Italian Seismic Bulletin (Bollettino Sismico Italiano, BSI). The results are published on the web, these are available to both the scientific community and the general public. The INGV surveillance includes a pre-operational tsunami alert service since INGV is one of the Tsunami Service providers of the North-eastern Atlantic and Mediterranean Tsunami warning System (NEAMTWS).

\section{Introduction}

The Istituto Nazionale di Geofisica e Vulcanologia (INGV) is an important Italian public research institution (http://www. ingv.it/en/); INGV carries on research in the Earth Sciences combining data from geophysical networks and results from fundamental research into the assessment of geo-hazards.

INGV is the reference institution of the Italian Department of Civil Protection (Dipartimento di Protezione Civile, DPC), and is part of the National Civil Protection System. It is in charge of the surveillance of the seismicity and volcanic activity of the entire national territory through stateof-the-art instrumental networks covering Italy and concentrated around the main active volcanoes.

The signals recorded by these networks are transmitted in real-time to the 24/7 operations rooms in Rome, Naples and Catania. The data are archived and distributed through web portal developed by INGV and integrated at European and global scales. 
INGV is also a constituent of the Italian tsunami warning system (Sistema Nazionale Allerta Maremoto, SiAM) and since recently tsunami service provides (TSP) of the NorthEastern Atlantic and Mediterranean Tsunami Warning System (NEAMTWS, next to operational, at the moment of writing this manuscript).

Here we describe the various networks, databases and monitoring products of the National Earthquake Center (Centro Nazionale Terremoti, CNT, one of the organizational units of INGV) and, concisely, the earthquake and tsunami surveillance systems at INGV Rome.

\section{National Seismic Networks}

INGV operates several permanent and mobile networks, deployed over the entire Italian territory. Such networks produce a large amount of observational data feeding both scientific and surveillance activities. The instrumental networks monitor all the relevant geophysical parameters for the $\mathrm{Na}$ tional and local Civil Protection systems and, at the same time, represent the foundation of virtually all research activities carried on by scientists to address the complex geodynamics of Italy and neighboring regions both locally and regionally. These networks comprise the main infrastructure managed by INGV, and focus the majority of its financial and human resources.

The CNT, has been in charge of developing and maintaining the national networks for the last 30 years, ensuring continuous technological update, increase of the quality and the quantity of the sensors and the improvement of data transmission and of the preprocessing performances.

The National Seismic Network (Rete Sismica Nazionale, RSN; Amato and Mele, 2008) counts about 400 state-ofart seismic stations equipped with 3-component velocimeters ranging from (very) broadband to short period (Fig. 1; http://cnt.rm.ingv.it/en/instruments).

The CNT, has installed and operates most of these stations, although a considerable number of stations that are also contributing to the INGV surveillance system has been installed and is maintained by other INGV offices (Naples, Catania, Bologna, Milan, Pisa, Arezzo). INGV network has international code IV (INGV Seismological Data Centre, 1997, Rete Sismica Nazionale (RSN), doi:10.13127/SD/X0FXNH7QFY; http://cnt.rm.ingv.it/en/instruments/network/IV). It is complemented by about $20 \mathrm{VBB}$ stations forming the Mediterranean Network (MedNet, international code $\mathrm{MN}$ ), managed by CNT in collaboration with the hosting Euro-Mediterranean Institutions (MedNet project partner institutions, Mediterranean Very Broadband Seismographic Network (MedNet) doi:10.13127/SD/FBBBTDTD6Q, http://cnt.rm.ingv.it/en/instruments/network/MN). Other Italian or European Institutions contribute to the RSN with their networks (http://cnt.rm.ingv.it/en/instruments).
The Italian institutions collaborating with INGV for the RSN include the Centro di Ricerche Sismologiche (Seismological Research Center, CRS) of the Istituto Nazionale di Oceanografia e di Geofisica Sperimentale - OGS (Italian National Institute for Oceanography and Experimental Geophysics) in Udine, "Prato Ricerche" in Prato, Osservatorio Sismico "Andrea Bina" in Perugia, several Universities: Genova Univ. (Dipartimento di Scienze della Terra, dell'Ambiente e della Vita, DISTAV, in Genova), Calabria Univ. (in Cosenza), Trieste Univ. (in Trieste) and some local municipalities as Bolzano and Trento. Monitoring earthquakes in the Euro - Mediterranean region and globally is carried out by collecting real-time global data, upon bilateral agreements, from many other European and extra-European stations through centers like the Helmholtz-Zentrum Potsdam - Deutsches GeoForschungsZentrum (GFZ), the United States Geological Survey and its National Earthquake Information Center (NEIC), Incorporated Research Institutions for Seismology (IRIS), National Observatory of Athens (NOA) and others.

Several of the seismic stations are also equipped with strong motion sensors (accelerometers) and with GPS antennas (Figs. 1, 2). The variety of instrumentation and data transmission in the RSN is detailed in Table 1. In particular, one of the digitizer widely adopted by INGV has been designed and is produced by CNT and is commonly referred as the GAIA data logger (Amato et al., 2006). This is a 24 bit Digital Acquisition System (DAS), that incorporates Linux Operating System and it is a modular, easily settable, low power and low cost DAS. It is quite compact and characterized by high connectivity.

The important technological upgrades carried out in the last years have allowed for significant improvements of the seismic monitoring of Italy and of the Euro-Mediterranean Countries. The adopted data transmission systems include satellite, local wireless connections (UMTS, WIFI etc.) and wired lines. Mainly Seedlink protocol has been adopted for data transmission.

Many of the sites of the RSN especially in southern Italy and Sicily are also GPS permanent recording sites of the "Rete Integrata Nazionale GPS" (INGV RING Working Group, Rete Integrata Nazionale GPS (RING) doi:10.13127/RING; Fig. 3). RING integrates GPS receivers with broad-band seismometers and accelerometers and the data are transmitted through a real-time satellite connection primarily to the main data hubs. Presently, we have installed more than 180 receivers connected with three main hubs at the acquisition centers located in Rome, Grottaminarda, and Catania. Nowadays, the RING represents a technologically advanced infrastructure and a reference point for several regional government institutions and enterprises in the field of the geodesy (Avallone et al., 2010).

The co-location of different instruments (Fig. 2) allows us to record the entire frequency range of the deformation process that leads to earthquake occurrence, from the inter- 


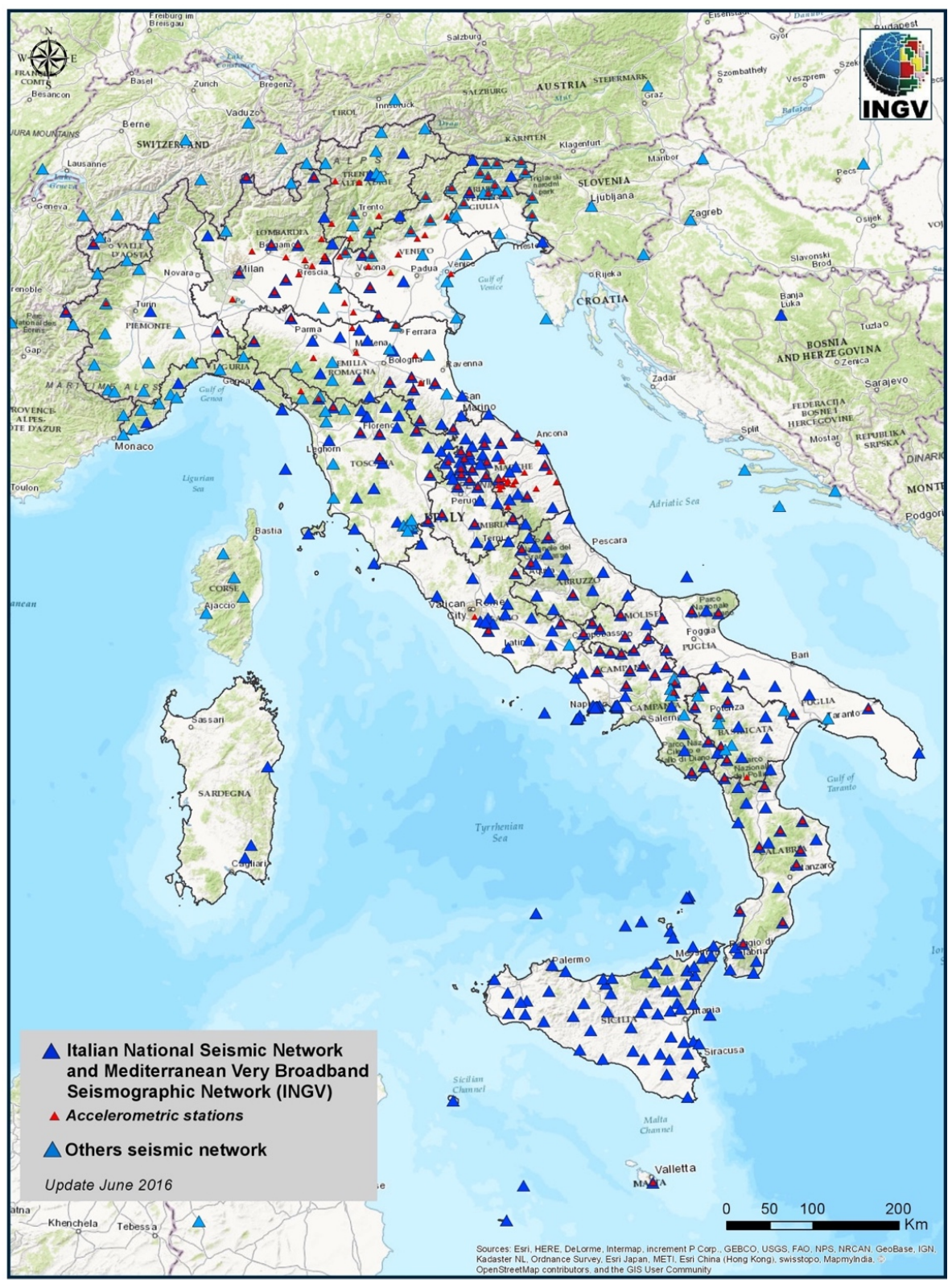

Figure 1. The Italian seismic network, RSN, dark blue triangles are the stations belonging to IV and MN networks directly owned and managed by INGV, light blue triangles are stations owned and managed by other national and international institutions that contribute to the INGV surveillance system. Red small triangles indicate stations equipped with accelerometers.

seismic strain accumulation on faults to the radiative part during the rupture process. Almost all the stations have been installed in the free field thank to the low power consumption of the satellite transmission that can be run with solar panels. Many RING GPS remote sites transmit data at $30 \mathrm{~s}$ of sampling rate in real-time streaming adopting the Nanometrics LIBRA VSAT technology and using the Intelsat and Hellasat satellite systems. The satellite communication for the transmission of the geodetic signal has been chosen for its autonomy, reliability, simplicity, possibility to have high quality of data and economy. As an alternative to the satellite systems some sites are provided with Internet communication (by cable, Universal Mobile Telecommunications System - UMTS, Satellite Internet) or dedicated WiFi Mesh Network designed, implemented and maintained by the CNT engineers and technicians. These infrastructures allow us to acquire the GPS data in real-time at $1 \mathrm{~s}$ of sampling rate. 

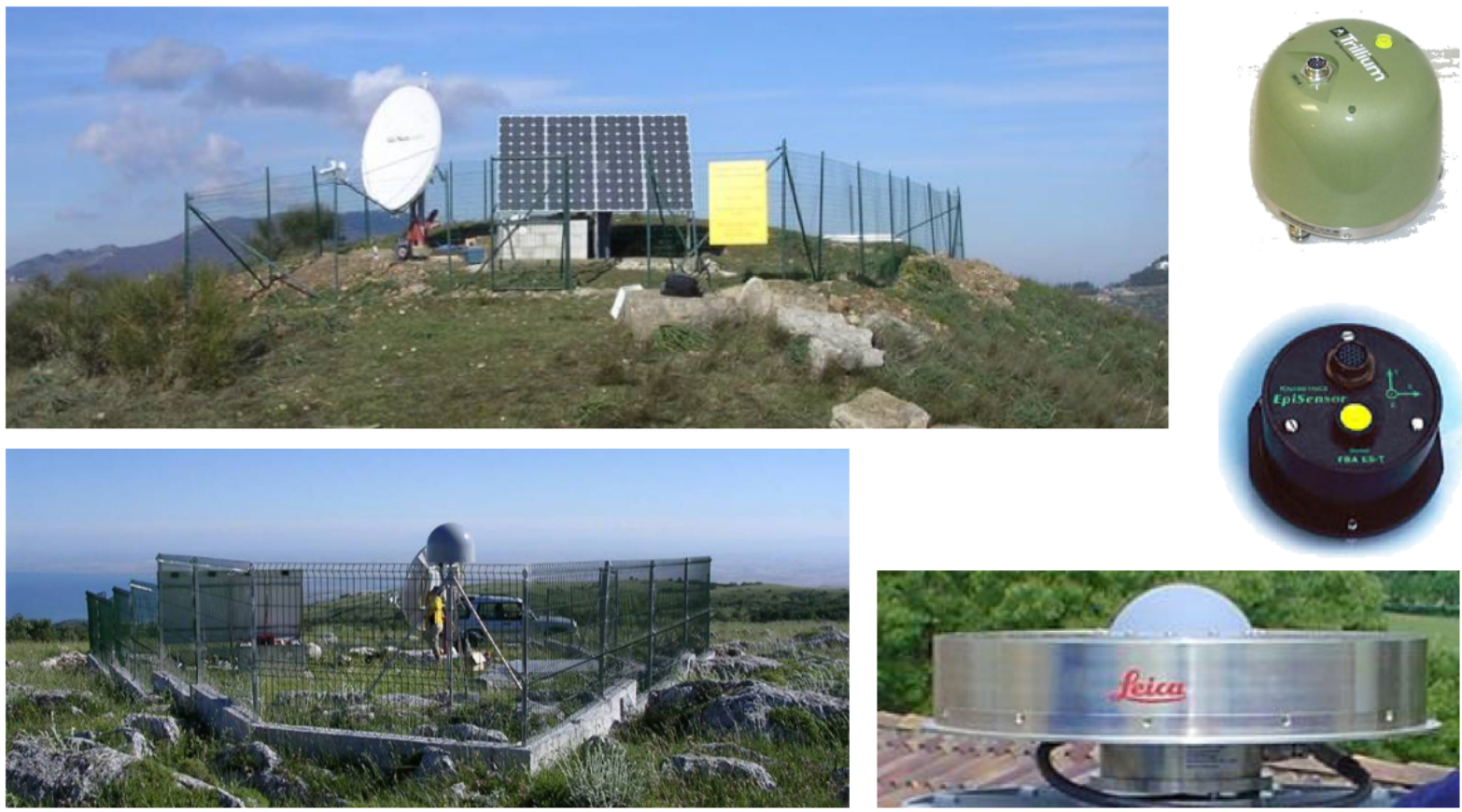

Figure 2. A site of the RSN-RING networks equipped with broad-band velocimeter, accelerometer and GPS antenna.

Table 1. Instrumentation - data transmission and data exchange protocols of RSN.

\begin{tabular}{llll}
\hline Sensors & Digitizers & Transmission & Data exchange protocols: \\
\hline Short period SP & GAIA & Cable & SeisComP, SEEDlink \\
Geotech S-13 & Trident/Cygnus & FASTWEB & Earthworm package SW \\
Lennartz LE-3D/20s & Taurus & RUPA & InterNAQS: Nanometrics \\
Kinemetrics SS-1 Ranger & HRD-24 & WIND & \\
Lennartz: LE-3D/1s borehole & Reftek 130 & INTERNET & \\
Malin borehole 2 Hz & Quanterra Q330 & Wireless & \\
Lennartz LE-3D/5s & Quanterra Q680 & WI-FI & \\
Lennartz LE-3D/1s & Quanterra Q4120 & UMTS & \\
Broad Band, Very BB BB - VBB & Quanterra Q730 & Satellite & \\
Guralp CMG 40 & Guralp DM-24 & HELLAS SAT & \\
Trillium-40 & & NANOMETRICS & \\
Trillium-120P & & INTELSAT & \\
Trillium-120compact & & SATLINK & \\
Trillium-240 & & Broadsat/Tooway & \\
Streckeisen STS-2 & & & \\
Streckeisen STS-1 & & & \\
Accelerometers & & & \\
Kinemetrics Episensor & & & \\
Colibrys SF3000 & & & \\
\hline
\end{tabular}

\section{Data archives and distribution}

The data acquired continuously from seismometric, accelerometric (Mazza et al., 2012) and GPS networks are archived at INGV following international standards. The distribution of the continuous waveforms (velocimeter and accelerometer signals) is done through the European Integrated seis- mic Data Archive (EIDA). EIDA is a distributed and federated data center part of ORFEUS (Observatories \& Research Facilities for European Seismology) established to securely archive seismic waveform data and related metadata, gathered by European research infrastructures, and to provide transparent access to the archives by the geosciences research communities (Fig. 4). INGV is one of the primary 


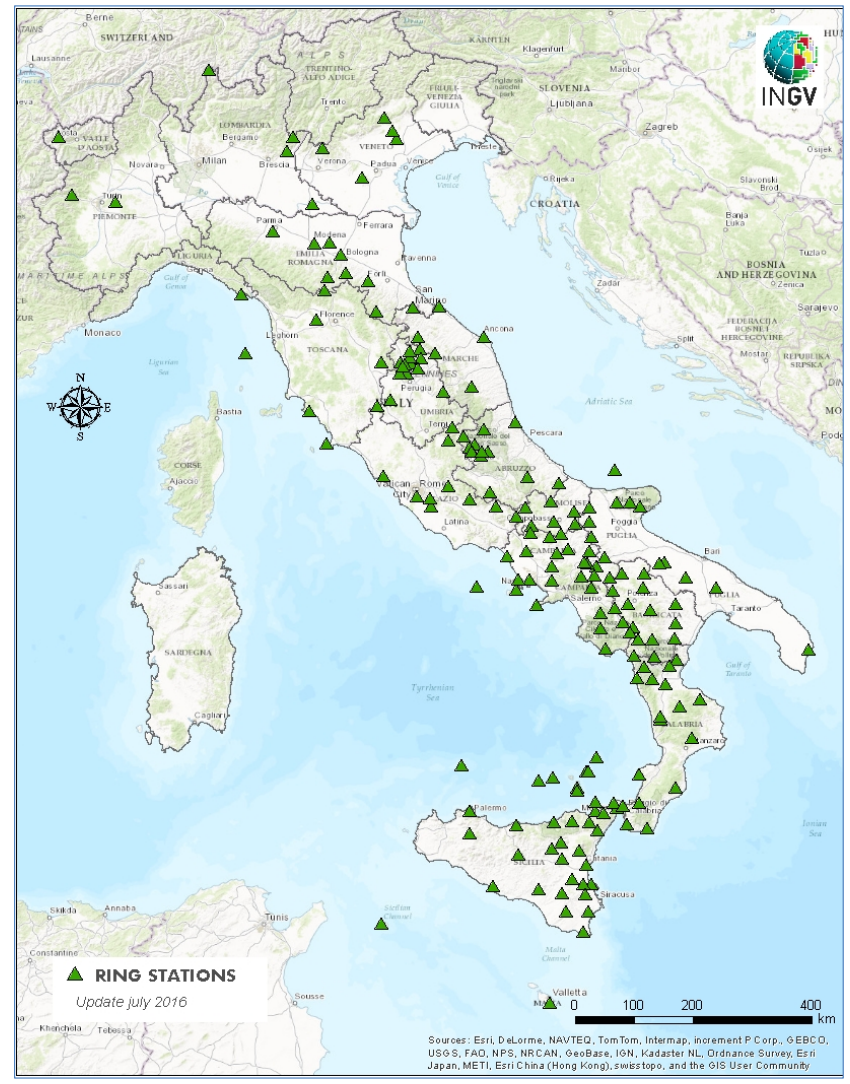

Figure 3. RING, the Italian GPS National network.

node of the federate archive, and develops and provides a series of web services to access, explore and download data and metadata contained in the archive (Fig. 5). EIDA nodes are data centers which collect and archive data from seismic networks. Networks contributing data to EIDA are listed in the ORFEUS EIDA network list (http://www.orfeus-eu. org/data/eida/networks/). Technically, EIDA is based on an underlying architecture developed by GFZ to provide transparent access to data of all nodes. Formally, EIDA is a special working group of ORFEUS - the organization that since 1987 started to promote digital seismology in Europe in all aspects, especially the research of seismic waves in a broad frequency range (so called "broad-band seismology").

The current EIDA nodes are: KNMI/ORFEUS Data Center, (ODC)/Netherlands, GEOFON/GFZ/Germany, SED/ETHZ/Switzerland, RESIF/INSU-CNRS/France, INGV/Italy, NIEP/Romania, KOERI/Turkey, BGR/Germany, NOA/Greece, IPGP/France and LMU/Germany. Other European institutions contribute with their data archives to EIDA in a continuous collaboration between institutions.

The seismometric and strong motion network data are also archived and distributed through event based archives as the Italian Seismological Instrumental and parametric Database (ISIDe working group, 2016), the Italian Acceleromet-

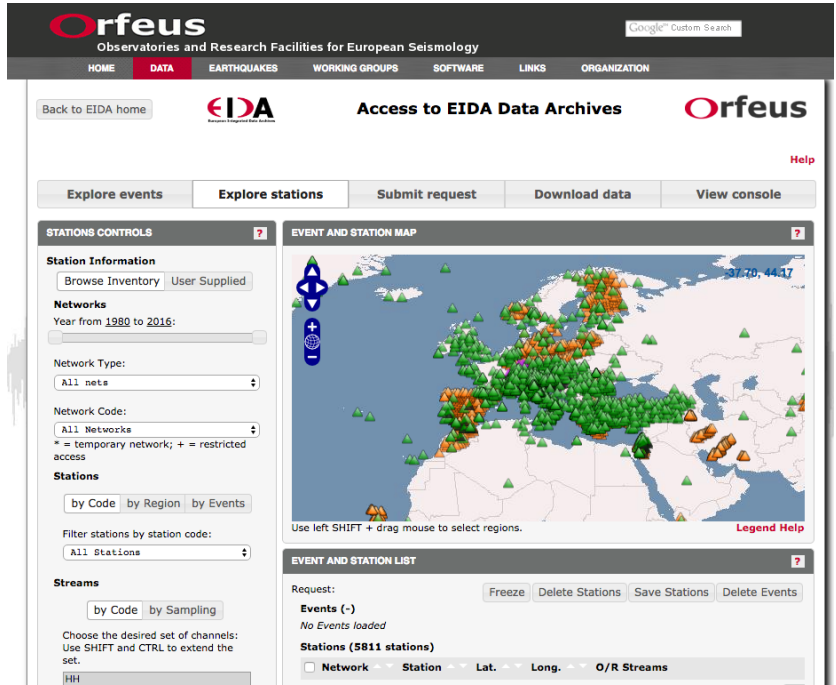

Figure 4. EIDA access portal to the seismic data.

ric Archive (ITACA working group, 2016), the Engineering Strong-Motion database (ESM working group, 2015) and INGV Strong Motion Data (ISMD, http://ismd.mi.ingv.it/). GPS data, from the RING network are archived, analysed and distributed at INGV, and can be found at http://ring.gm. ingv.it/ (INGV RING Working Group, 2016).

GPS data, from the RING network are archived, analyzed and distributed at INGV, and can be found at http://ring.gm. ingv.it/.

\section{Operations}

The INGV seismic monitoring system provides prompt and reliable earthquake locations and magnitudes occurring in Italy and surrounding areas (Amato and Mele, 2008), and accurate estimates in the Mediterranean Basin and worldwide (Bernardi et al., 2015). The data recorded by the monitoring networks are transmitted in real-time to the operational rooms in Rome, Naples and Catania, where highly trained 24/7 staff analyze them to obtain the main parameters of the ongoing events and processes. Additional disasterrecovery rooms in Rome and Grottaminarda can be activated if needed. Monitoring products are the basis of the seismic surveillance and tsunami alert done by INGV as a part of the National Civil Protection System coordinated by the Italian DPC. Since October 2014 a pre-operational service of tsunami alert for the Mediterranean Sea regions started operating in the same operational room in Rome where seismic surveillance is carried out. 


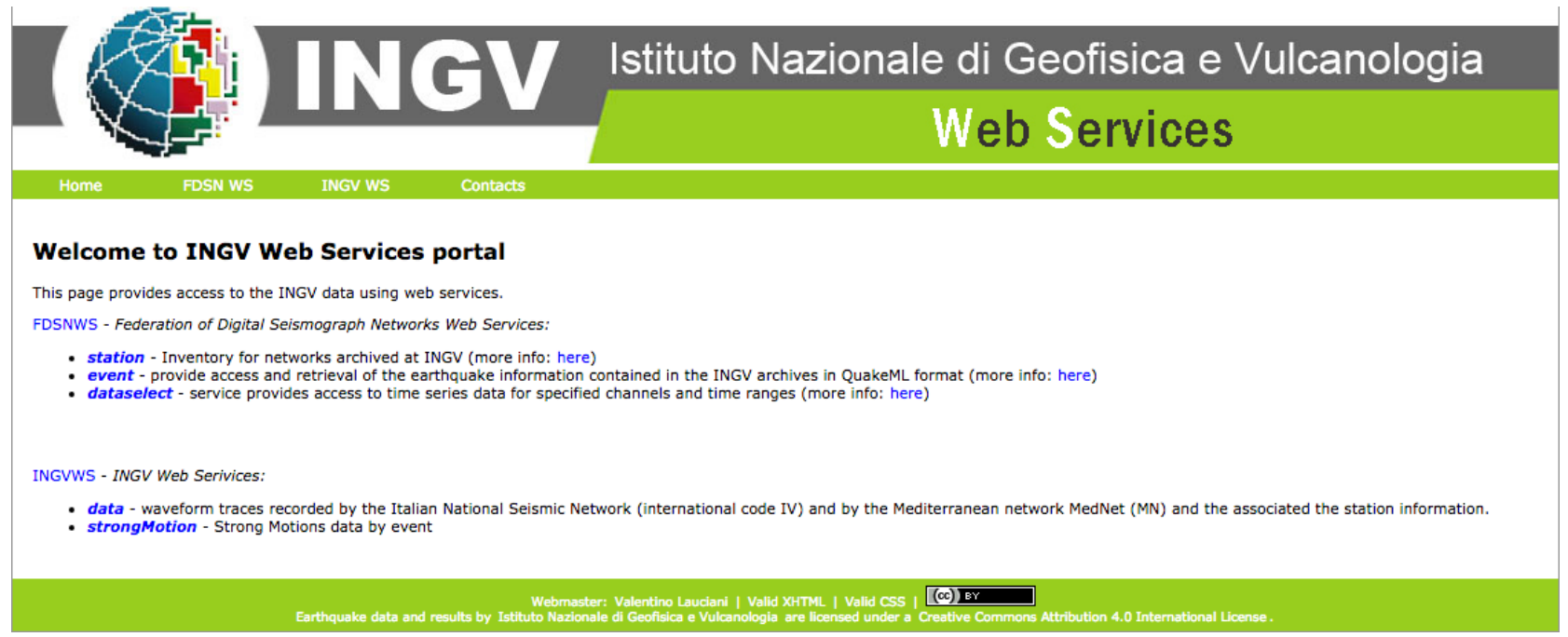

Figure 5. INGV web services at http://webservices.rm.ingv.it/.

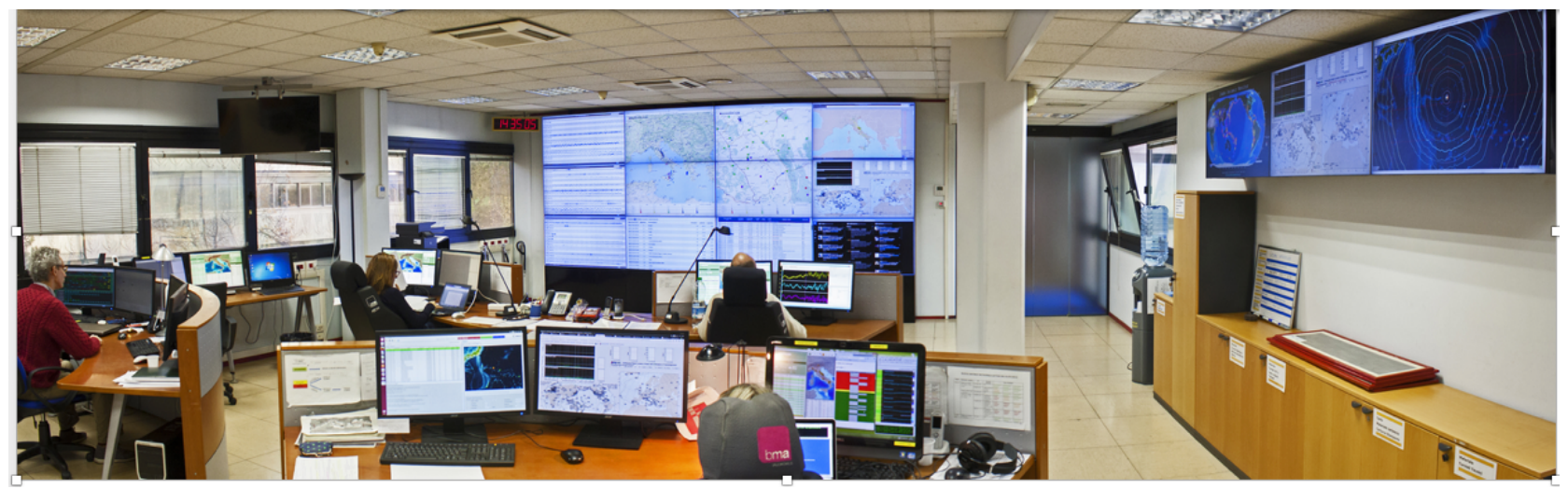

Figure 6. Surveillance, operational room in Rome.

\subsection{Seismic surveillance}

The seismic data flow continuously, with maximum delays of a few seconds, into the INGV acquisition system in Rome, where several automatic procedures, availing of the Earthworm software package (Mele et al., 2010 and references therein) allow for the near real-time detection of local and distant earthquakes, their locations and magnitudes and the creation of reports and maps (including shake maps, e.g. http://cnt.rm.ingv.it/en/event/7073641, tab Impact) for the DPC. For earthquakes in Italy the current agreement between INGV and DPC states that the first information with location and magnitude must be sent within the first $120 \mathrm{~s}$ after any potentially felt earthquake in Italy. More detailed information follows in the next few minutes (typically 10 to $15 \mathrm{~min}$ ), during which seismologists on the duty shift revise earthquake location and magnitude. The total number of people involved in the shifts at the operational center consists of about 120 persons, including researchers, technologists and technical staff. The operations in the seismic and alert center are organized in three shifts of four persons (two seismologists, a tsunami specialist and an engineer) per day. In case of need, i.e. earthquakes above a certain threshold, additional technical people and earthquake or tsunami experts become available and reach the center.

Earthquakes from abroad are also examined and promptly notified to the DPC (i.e., $M \geq 5.0$ for the Euro-Mediterranean region and $M \geq 6.0$ globally). Above certain magnitude threshold or upon DPC request, an earthquake report is produced in the first 1-2 $\mathrm{h}$ after the origin time.

As an example of the operational activities, during 2014 more than 27000 earthquakes have been located in Italy and surrounding areas. Slightly less than 800 earthquakes have been notified according to the procedure outlined above to DPC (i.e., $M \geq 2.5$ ) via phone, e-mail, sms, and published in quasi real-time on the web and on social networks (e.g., 
http://cnt.rm.ingv.it; wordpress, google+, twitter, app IOS by Apple and Facebook).

Similarly, notifications regarding 96 global earthquakes above 6.0 in magnitude have been produced.

Earthquake parameters of the events recorded by the RSN and located by the INGV monitoring center in Rome, are readily available on the web page http://cnt.rm.ingv.it/ and in ISIDe. On a routine basis, the analysts of the Italian Seismic Bulletin (Bollettino Sismico Italiano, BSI) double check the parameters of all the recorded earthquakes by placing weights and polarity of arrivals of seismic waves and integrating all the data available in the acquisition system. Starting from 2015, BSI is published every 4 months on the web page http://cnt.rm.ingv.it/bsi and is available for download in QuakeML format. For each release of the BSI, is also possible to download a technical document (in Italian, pdf format) which describes the main features of the Italian seismicity and seismic network and specifies the BSI working group. A DOI is assigned to each BSI release to make easily quotable the work done by the BSI group (i.e. doi:10.13127/BOLLETTINO/2016.01).

\subsection{Tsunami alert}

In 2013, the Tsunami Warning Centre (Centro Allerta Tsunami, CAT) was established at the INGV headquarters in Rome. CAT is an internal structure of INGV with the tasks of developing the Italian Tsunami Service Provider (TSP) and of assessing the National Tsunami Hazard Map. In 2014, the required software and the data acquisition systems were prototyped, engineered and implemented. Concurrently, about 80 persons, among researchers and technicians, have been trained to fulfill the new 24/7 tsunami-expert duty shift. On 1 October 2014, INGV started operating as cTSP within the NEAMTWS framework in pre-operational mode for its competence zone (the whole Mediterranean Sea from Gibraltar to the Marmara Sea included) and it has been very recently approved as TSP. The Italian-TSP is located in the "seismic monitoring center" at the INGV headquarters in Rome and it benefits of the existing physical infrastructure and human resources. In fact, the personnel trained for Tsunami alert participate also to the seismic surveillance shifts and the operations of the Italian-TSP have been integrated with those of seismic surveillance that INGV provides to DPC.

INGV receives real-time seismic data, recorded by about 400 stations distributed worldwide, through several institutions (GFZ, NOA, CENtre d'Alerte aux Tsunamis-CENALT, IRIS, NEIC, INGV). Seismic data are continuously and automatically analyzed by Early-Est, a software jointly developed by ALomax Scientific and INGV (Bernardi et al., 2015 and references therein; http://early-est.rm.ingv.it/warning. html) that performs seismic detection, location and magnitude estimation. Once an earthquake is detected, a software procedure is triggered automatically to produce, depending on earthquake hypocenter and magnitude, a tsunami alert message that personnel on duty swiftly verifies and then delivers to a pre-defined list of recipients (i.e., Countries that subscribed the service, DPC, international institutions and to the other cTSPs and TSPs).

INGV also receives in (quasi) real-time sea level data recorded by hundreds of tide-gauges distributed worldwide through the Sea Level Monitoring Facility of IOC. In addition, the Istituto Superiore per la Protezione e la Ricerca Ambientale (ISPRA, the institution managing and operating the Italian National Mareographic Network) sends to INGV the sea level data of their stations distributed along the coast of Italy. These data can be analyzed by the tsunami expert on duty to confirm (or cancel) the tsunami alert messages issued previously.

Since the end of 2014 several hundreds of $M 5.5+$ have been analyzed on the global scale, along with assessment of their tsunamigenic potential. This activity is performed in training mode with messages and reports distributed only internally, to warrant through continuous monitoring readiness and efficiency in case of an event in the Euro-Mediterranean area. For the most important events, such as the $8.3 M_{\mathrm{W}}$ (16 September 2015, 22:54:33 UTC) Coquimbo earthquake in Chile and the following tsunamis, further reports including tsunami simulations have been produced and both sent to DPC and published on the INGV website.

\section{Conclusive remarks}

The Italian national seismic network is part of the earthquake and tsunami monitoring and surveillance systems of the INGV. An efficient and rapid real-time monitoring system, producing epicenter, Ml, shake maps etc., is fundamental for the Italian DPC to lead the rescue teams quickly to the right place and reduce the number of fatalities in earthquake disasters. The monitoring networks are also powerful tools for scientific research. At international level (European and Global) real-time data sharing among countries is improving as well as the collaboration between the various European and International centers. INGV is a fundamental partner in two European projects: EPOS (European Plate Observing System; https://www.epos-ip.org/) currently in the implementation phase, aims at creating a pan-European infrastructure for solid Earth science to support a safe and sustainable society, and ARISTOTLE Project (All Risk Integrated System TOwards Trans-boundary hoListic Early-warning; http://aristotle.ingv.it/) just started in 2016 for providing expert scientific judgment to the Emergency Response Coordination Centre (ERCC) for its prompt response to meteo and geo natural disasters.

\section{Data availability}

The work presented in this article does not rely on any specific data sets. Rather this article presents primarily the ac- 
tivities of the INGV "Centro Nazionale Terremoti" responsible for the acquisition and distribution of several data sets through several databases that are either property of INGV or they are federated to. All the relevant sources of data are either cited in the text or accessible through the proper DOI in the references. We note that some DBs include not only INGV data but also data from other networks as it is detailed in the associated citations (e.g., ESM working group, 2015; ITACA working group, 2016; INGV RING Working Group, 2016) or cited websites (e.g., http://www.orfeus-eu.org/data/ eida/networks/).

Acknowledgements. This article follows from the presentation given at EGU 2016, session "Improving seismic networks performances: from site selection to data integration" and the authorship therein. In addition, we thank all INGV staff (technicians, analysts, people on duty for the seismic and tsunami surveillance, colleagues from the different INGV offices), that in the last years deployed seismic stations, analyzed seismic data and developed the Italian monitoring and surveillance system. In particular, we thank Fabrizio Bernardi, Aladino Govoni, Stefano Lorito, Alessio Piatanesi and Fabrizio Romano for their fundamental contribution to the development of the tsunami alert system at INGV. This study was funded by the Italian Presidenza del Consiglio dei Ministri Dipartimento della Protezione Civile (DPC). This paper does not necessarily represent DPC official opinion and policies.

Edited by: D. Pesaresi

Reviewed by: I. Bondar, M. Popa, and one anonymous referee

\section{References}

Amato, A. and Mele, F. M.: Performance of the INGV National Seismic Network from 1997 to 2007, Annals of Geophysics, 51, 417-431, doi:10.4401/ag-4454, 2008.
Amato, A., Badiali, L., Cattaneo, M., Delladio, A., Doumaz, F., and Mele, F. M.: The real-time earthquake monitoring system in Italy, Géosciences - Révue du BRGM, No. 4, 2006, http://hdl.handle. net/2122/2574 (last access: 20 October 2016), 2006.

Avallone, A., Selvaggi, G., D’Anastasio, E., D’Agostino, N., Pietrantonio, G., Riguzzi, F., Serpelloni, E., Anzidei, M., Casula, G., Cecere, G., D’Ambrosio, C., De Martino, P., Devoti, R., Falco, L., Mattia, M., Rossi, M., Obrizzo, F., Tammaro, U., and Zarrilli, L.: The RING network: improvement of a GPS velocity field in Central Mediterranean, Annals of Geophysics, 53, doi:10.4401/ag-4549, 2010.

Bernardi, F., Lomax, A., Michelini, A., Lauciani, V., Piatanesi, A., and Lorito, S.: Appraising the Early-est earthquake monitoring system for tsunami alerting at the Italian Candidate Tsunami Service Provider, Nat. Hazards Earth Syst. Sci., 15, 2019-2036, doi:10.5194/nhess-15-2019-2015, 2015.

ESM working group: Engineering Strong-Motion database, version 1.0, doi:10.13127/ESM, 2015

INGV RING Working Group: Rete Integrata Nazionale GPS (RING) [Data set]. Istituto Nazionale di Geofisica e Vulcanologia (INGV), doi:10.13127/RING, 2016.

INGV Seismological Data Centre: Rete Sismica Nazionale (RSN), Istituto Nazionale di Geofisica e Vulcanologia (INGV), Italy, doi:10.13127/SD/X0FXnH7QfY, 1997.

ISIDe working group: Italian Seismological Instrumental and parametric Data-base, version 1.0, doi:10.13127/ISIDe, 2016.

ITACA working group: ITalian ACcelerometric Archive, version 2.1, doi:10.13127/ITACA/2.1, 2016.

Mazza, S., Basili, A., Andrea Bono, Valentino Lauciani, Mandiello, A. G., Marcocci, C., Mele, F. M., Pintore, S., Quintiliani, M., Scognamiglio, L., and Selvaggi, G.: AIDA - Seismic data acquisition, processing, storage and distribution at the National Earthquake Center, INGV Annals of Geophysics, 55, 54-548, doi:10.4401/ag-6145, 2012.

Mele, F. M., Bono, A., Lauciani, V., Mandiello, A., Marcocci, C., Pintore, S., Quintiliani, M., Scognamiglio, L., and Mazza, S.: Tuning an earthworm phase picker: some considerations on the pick_ew parameters, Rapporti tecnci INGV, no. 164, 2010. 